\title{
Plasma Renin Activity and the Response to Sodium Depletion in Salt-Losing Congenital Adrenal Hyperplasia
}

\author{
BRUCE S. KEENAN, ${ }^{(25)}$ JOHN H. HOLCOMBE, ${ }^{(27)}$ DONNIE P. WILSON, ${ }^{(28)}$ \\ REBECCA T. KIRKLAND, ELAINE POTTS, ${ }^{(29)}$ AND GEORGE W. CLAYTON \\ Department of Pediatrics, Baylor College of Medicine, Houston, Texas, USA
}

\section{Summary}

Mineralocorticoid therapy was discontinued in 14 subjects, ages 7-17 years, with salt-losing congenital adrenal hyperplasia. The level of plasma renin activity (PRA) was determined in relation to the cumulative loss of sodium (intake-urinary output) over 4-12 days. Although the magnitude and rate of response varied, PRA increased in all subjects. There was a significant positive correlation between cumulative sodium loss and PRA $(r=0.585, P<$ $0.001, n=76$ ), and a significant negative correlation between serum sodium concentration and PRA $(r=-0.736, P<0.001, n$ $=76$ ). Significant sodium loss and elevation of PRA were seen prior to the development of hyponatremia. Thus, elevated PRA appeared to be a more sensitive indicator of sodium loss than serum sodium concentration.

Fifteen infants, ages 1-33 months with salt-losing congenital adrenal hyperplasia, who were treated with subcutaneous pellets of 11-deoxycorticosterone acetate (DOCA), were also studied. PRA decreased following implantation of the DOCA pellets over 1-6 months. In most instances there was a subsequent increase in PRA, beginning at 1-8 months post implantation. There was a significant negative correlation between serum sodium and PRA $(r=-0.669, P<0.001, n=78)$ in these infants. However, there were four instances in which elevated PRA was associated with normal serum sodium.

Sodium balance studies were also performed on 14 of these infants during 29 admissions. All balance studies were performed at approximately 3 or 6 months after DOCA pellet implantation. On a diet containing $2 \mathrm{mEq} \mathrm{Na/kg}$ body weight per day, sodium balance was positive and there was no significant change in sodium excretion over the 3 days of observation. When a $1 \mathrm{mEq} \mathrm{Na} / \mathrm{kg} /$ day diet was given, sodium balance was neutral on day 3 of the study, and fractional urinary sodium excretion $\left(\mathrm{Fe}_{\mathrm{Na}}\right)$ decreased from $0.49 \pm 0.33$, S.D. on day 1 to $0.23 \pm .09 \%$ on day $3(P<$ $0.001, n=19$ ). This change was statistically significant. Net sodium balance on either diet was independent of PRA. Elevated PRA was not necessarily associated with adverse symptomatology.

Thus in infants treated with DOCA pellets, elevated PRA was associated with indices of sodium depletion and decreased availability of DOCA. The dietary sodium requirement for infants treated with DOCA appeared to be 1-2 $\mathrm{mEq} / \mathrm{kg}$ body weight/day.

\section{Speculation}

Although increased PRA indicated the subject's response to sodium depletion, it did not predict the ability to maintain sodium balance. There must be factors other than mineralocorticoid therapy, some possibly renin-dependent, contributing to the ability to compensate for sodium depletion. Nevertheless, increased PRA may be taken as an indication to either increase mineralocorticoid dosage or sodium intake.

Salt-losing congenital adrenal hyperplasia (SL-CAH) is an inherited defect in steroid C 21-hydroxylation, characterized by deficiency in the secretion of cortisol, hypersecretion of androgens and progestins $(2)$, and complete $(3,12)$ or partial $(6,10)$ defects in the synthesis of aldosterone. Salt-wasting in this disorder appears to be the result both of defective aldosterone synthesis and of increased production of steroids which antagonize the effects of aldosterone $(9,10)$. Glucocorticoid replacement normally consists of cortisone acetate, given intramuscularly or orally. Parameters of androgen and progestin $(11,21)$ secretion have been used to assess the adequacy of ACTH suppression by the administered glucocorticoid. Therapy of salt wasting in infants consists of dietary sodium chloride supplementation and the administration of a mineralocorticoid, either parenteral DOCA or oral $9 \alpha$-fluorohydrocortisone $(9 \alpha \mathrm{FF})$. In older subjects oral $9 \alpha \mathrm{FF}$ is usually given.

In the past, assessment of the adequacy of mineralocorticoid therapy has consisted of the estimation of growth, blood pressure, serum electrolytes, and the presence or absence of signs and symptoms of sodium depletion $(21,23)$. More recently, measurement of PRA has been suggested as a laboratory aid in the management of sodium wasting $(7,14)$. However, PRA may be elevated in the non salt-losing form of CAH (18), and no systematic study has been published concerning the relationship of PRA to parameters of sodium homeostasis. The present study examined those parameters in infants and older subjects with CAH. Our data indicate that elevated PRA reflects sodium depletion in this disease, but surprisingly did not predict the subject's ability to maintain sodium balance.

\section{MATERIALS AND METHODS}

\section{SUBJECTS}

The subjects of the study all had salt-losing $\mathrm{CAH}$ due to 21 hydroxylase deficiency. In the 15 infants, age 1-33 months (10 females, 5 males), the diagnosis was made by demonstration of elevated urinary 17-ketosteroids and plasma 17-hydroxyprogesterone. In a previous report concerning these infants, increasing levels of plasma 17-hydroxyprogesterone were demonstrated over the first $3 \mathrm{wk}$ of life, sometimes associated with increased aldosterone levels (5). In the older subjects, age 7-17 years, the diagnosis had been made by demonstration of elevated urinary 17-ketosteroids and either pregnanetriol or 11-ketopregnanetriol. Salt-losing was documented by hyponatremia, hyperkalemia, and increased urinary sodium excretion in infancy.

\section{STUDIES IN OLDER SUBJECTS}

Prior to study, all were being treated with cortisone acetate 20 $35 \mathrm{mg} / \mathrm{m}^{2}$ body surface area per day in 3 divided doses and $9 \alpha \mathrm{FF}$ $0.05-0.2 \mathrm{mg} /$ day. All had supine PRA values of $1.3-8 \mathrm{ng} / \mathrm{ml} / \mathrm{liter}$ initially. Fourteen subjects ( 7 female, 7 male) were admitted to the Clinical Research Center, Texas Children's Hospital and given a diet constant in sodium content, 2.5 times the estimated normal 
maintenance amount for 4 days followed by 4 days of a diet containing 0.8 times maintenance sodium. Normal maintenance sodium requirement was $2.5 \mathrm{mEq} / 100 \mathrm{kcal}$ metabolized (22). Sodium intake ranged from $2.5-4.5 \mathrm{mEq} / \mathrm{kg}$ /day at 2.5 maintenance and from 0.75 to 1.40 at 0.8 maintenance. Therapy with $9 \alpha \mathrm{FF}$ was discontinued at the beginning of the balance studies. Glucocorticoid therapy was maintained by the substitution of dexamethasone, $1.5-2 \mathrm{mg} / \mathrm{m}^{2} /$ day in 3 divided doses. Urinary sodium excretion, serum sodium and PRA (supine overnight) were measured daily. The study was discontinued after 8 days or when decrease in serum sodium or the onset of symptoms of salt-wasting indicated the need for an earlier termination.

\section{STUDIES IN INFANTS}

The infants were receiving therapy with cortisone acetate $25-50$ $\mathrm{mg}$, intramuscularly, every 3 days and had received one or two pellets of DOCA, implanted subcutaneously. Most infants received subcutaneous DOCA therapy approximately every 6 months for the first 24-36 months of life. Blood samples were obtained for determination of serum sodium and PRA at various times after implantation of pellet(s). No attempt was made to control posture in the infants. In all, 31 pellet cycles in 15 infants were studied. On 29 occasions, these infants were admitted to the Children's Clinical Research Center for studies of sodium balance at 2-6 months post pellet implantation. During those admissions an initial blood sample for PRA was obtained, and daily blood samples were obtained for serum sodium and creatinine determinations. A diet constant in sodium content was given for 3 days, either 1.0 or $2.0 \mathrm{mEq} / \mathrm{kg}$ body weight/day. Urine collections were made for measurement of sodium and creatinine excretion. At the conclusion of the study a blood sample for PRA was obtained.

\section{METHODS}

PRA determinations used two methods: the older subjects were studied between 1975 and 1978 and The New England Nuclear Kit was used (15). Normal values on an ad libitum diet: supine $0.5-1.6 \mathrm{ng} / \mathrm{ml} / \mathrm{h}$ angiotensin $I$ and upright 1.9-3.7. The infants were studied between 1977 and 1981 and the method of Sealy, et al. was used (19). Normal values: for infants 0-6 months 6.7-41.6 $\mathrm{ng} / \mathrm{ml} / \mathrm{h}$ angiotensin I (posture not controlled), and for older children and adults; supine 0-8.0. Plasma samples were assayed by both methods in 33 instances. The range of values was from $0.1-116$ by the old method and $0.5-143$ by the new method. The values gave a linear correlation coefficient of 0.959 . The slope of the correlation line was 1.23 (Sealy method/New England Nuclear Kit method). Sodium and creatinine were measured by routine methods.

\section{CALCULATIONS}

Sodium balance was estimated as intake minus urinary excretion. Cumulative sodium loss was the cumulative sum of the daily difference, intake - urinary excretion. Fractional urinary sodium excretion $\left(\mathrm{Fe}_{\mathrm{Na}}\right.$ ) was calculated as follows:

$$
\mathrm{Fe}_{\mathrm{Na}}(\%)=\frac{[\mathrm{Na}]_{\mathrm{u}}[\mathrm{Cr}]_{\mathrm{s}}}{[\mathrm{Na}]_{\mathrm{s}}[\mathrm{Cr}]_{\mathrm{u}}} \times 100,
$$

where $[\mathrm{Na}]$ and $[\mathrm{Cr}]$ are concentrations of sodium and creatinine. The subscripts $u$ and $s$ refer to urine and serum, respectively. In order to have a defined starting point for sodium depletion in the older subjects, those in whom a supine PRA value between 1.5 and 8 was obtained early in the course of an admission, were used for this study. In several instances, when PRA was $<1$ initially, the point in the admission at which supine PRA increased to $>1.5$ was taken as the starting point. This limited he study to those subjects who, initially, were neither volume expanded (suppressed renin) nor severely volume contracted (markedly elevated renin). Cumulative sodium balance was calculated using that day as day 1. The means \pm S.E. of all PRA values in a given interval of cumulative sodium loss, $0-1.99 \mathrm{mEq} / \mathrm{kg} / \mathrm{kg}, 2.0-3.99$, etc., were used to define the relationship of these two parameters (Fig. 1).

Linear correlation coefficients $(r)$ were computed on a HewlittPackard HP-91 calculator. The statistical significance of the correlation coefficients was assessed by determining the probability that the slope of the regression line was different from zero. The value $r^{2}$ was taken to indicate the fraction of the variance of the dependent variable which was determined by the variance of a given independent variable (20).

\section{RESULTS}

The response to withdrawal of mineralocorticoid therapy in older CAH subjects is seen in Figure 1. In this group, the initial supine PRA values were slightly elevated $(3.2 \pm 1.4$ S.D. $\mathrm{ng} / \mathrm{ml}$ / h) and serum sodium concentrations were normal $(140 \pm 2.2$ $\mathrm{mEq} /$ liter). Progressive sodium depletion (cumulative sodium loss) occurred in all subjects, over 4-12 days, associated with increasing PRA and decreasing serum sodium, but there was considerable individual variation in the rate of increase of PRA in relation to the degree of sodium loss. Linear correlation between cumulative sodium loss and PRA was highly significant: $r=0.585$, $P<0.001, n=76$. Likewise there was a high degree of correlation between serum sodium and PRA: $r=-0.736, P<0.001, n=76$.

Significant sodium depletion and hyperreninemia occurred before hyponatremia was seen. Thus, mean cumulative sodium loss was $6-8 \mathrm{mEq} / \mathrm{kg}$ when serum $\mathrm{Na}$ had decreased to $135 \mathrm{mEq} /$ liter. This change occurred over 2-8 days. In three subjects, who had experienced sodium loses of $8.5-11.2 \mathrm{mEq} / \mathrm{kg}$ with PRA values of 44-80 $\mathrm{ng} / \mathrm{ml} / \mathrm{h}$, serum sodium remained in the normal range. PRA values greater than 15 were seen in 10 of 14 subjects prior to the development of hyponatremia $(\mathrm{Na}<135)$.

The demonstration of a clear relationship between PRA and
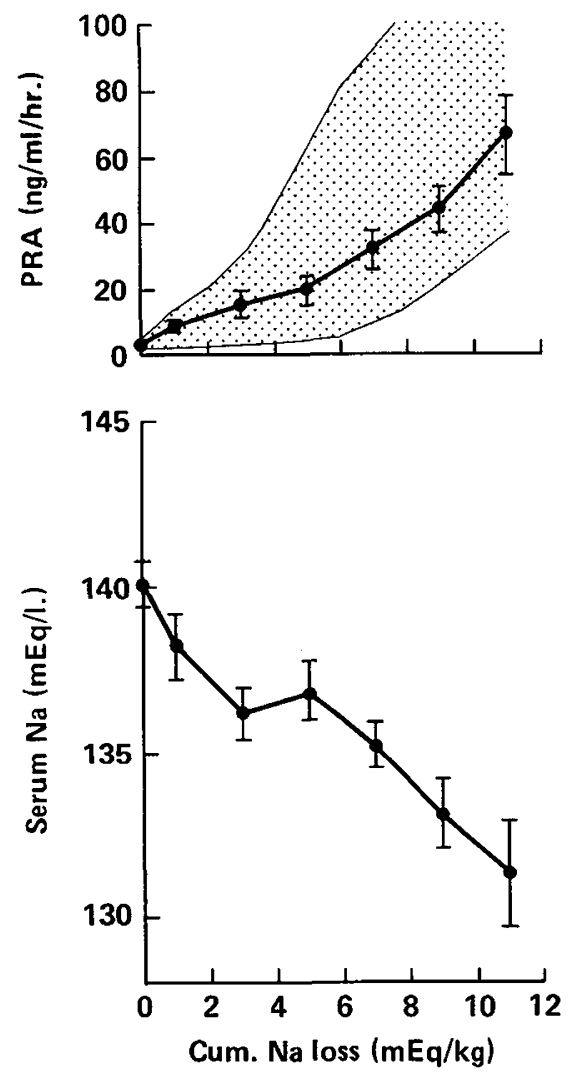

Fig. 1. Changes in PRA and serum sodium concentration in response to sodium depletion in SL-CAH subjects following discontinuation of therapy with $9 \alpha \mathrm{FF}$. The values of PRA and $\mathrm{Na}$ are mean \pm S.E., within intervals of $\pm 1 \mathrm{mEg} / \mathrm{kg}$ of cumulative sodium loss. The shaded zone in the upper panel is the range of observations. 
sodium depletion in the untreated older subjects, lead to the study of infants treated with subcutaneous DOCA pellets. In these subjects the source of mineralocorticoid was considered to be constantly diminishing, as the DOCA pellets were resorbed. As seen in Figure 2, PRA levels declined during the first few months after pellet implantation (30). Subsequently, PRA levels increased, but the time course was highly variable. These observations were true for initial therapy, which consisted of two $125 \mathrm{mg}$ DOCA pellets and 3-5 gm/day sodium chloride added to the diet for 1-3 months, and subsequent therapy which consisted of single $125 \mathrm{mg}$ DOCA pellets and an ad libitum diet. The infants' linear growth, weight gain, blood pressure and symptoms of salt-wasting (irritability, anorexia, vomiting and constipation) did not correlate with PRA. On four occasions those parameters, as well as serum sodium were normal, but PRA was greater than 40, the upper limit for normal breast-fed infants. As was true in the older subjects, decreasing serum sodium was associated with increasing PRA (Fig. 3). However, in 14 of 26 instances in which PRA was significantly elevated $(>40)$, serum sodium was in the normal range.

The ability of these infants to conserve sodium at 3-6 months after pellet insertion was tested by placing them on constant sodium intake for 3 days and measuring sodium balance and $\mathrm{Fe}_{\mathrm{Na}}$. On day 3 of the $2 \mathrm{mEq} / \mathrm{kg} /$ day diet, body weight was stable and mean sodium balance was positive, $0.56 \mathrm{mEq} / \mathrm{kg} /$ day \pm 0.30 S.D. $\mathrm{Fe}_{\mathrm{Na}}$, which did not change significantly during these studies, was $0.40 \% \pm 0.08$ S.D., $n=10$. When the infants were fed $1 \mathrm{mEq} \mathrm{Na}$ / $\mathrm{kg} /$ day, $\mathrm{Fe}_{\mathrm{Na}}$ decreased from $0.49 \pm 33$ to $0.23 \pm 0.09 \%, n=19$, $(P<0.001$ by paired $t$ test). The measured sodium balance was barely positive, $0.01 \pm 0.31$. Since stool and sweat loses were not measured, the majority of infants receiving the $1 \mathrm{mEq} \mathrm{Na} / \mathrm{kg} /$ day diet were considered to have been in negative sodium balance. Mean weight loss for this group was $2.0 \%$.

In the infants, the PRA on day 1 of the study was compared to $\mathrm{Fe}_{\mathrm{Na}}$ and to sodium balance on day 3 (Fig. 4). The range of initial
PRA was $1-143 \mathrm{ng} / \mathrm{ml} / \mathrm{h}$, but the initial serum sodium concentrations were normal for all subjects. Sodium balance and $\mathrm{Fe}_{\mathrm{Na}}$ on day 3 did not vary with PRA on the $2 \mathrm{mEq} / \mathrm{kg} /$ day diet. When 1 $\mathrm{mEq} \mathrm{Na} / \mathrm{kg} /$ day was given, a slight decline in sodium balance was associated with increasing PRA, but this was not statistically significant. In any event, the value of $r^{2}, 0.10$, indicated that no more than $10 \%$ of the variance in sodium balance was determined by PRA.

\section{DISCUSSION}

There is general agreement that PRA is increased in untreated salt-losing $\mathrm{CAH}(6,14,18)$ and that mineralocorticoid therapy results in lowering of these values $(6,14,8)$. In the present study, a highly significant correlation was demonstrated between sodium depletion or serum sodium concentrations and PRA. This was true for subacute sodium depletion in the older subjects and for chronic studies in infants. The $\mathrm{r}^{2}$ values indicated that as much as $55 \%$ of the variation in PRA could be accounted for by changes in these parameters of sodium depletion.

Other factors which may have influenced the PRA values, such as blood volume, renal blood flow, catecholamine secretion, and serum potassium concentration were not considered in this analysis. In addition, the contribution of renin secretion to sodium homeostasis, which might be expected to compensate for sodium depletion in these subjects, was not determined. Secretion of renin by the kidney is only the first step in the sequence leading to increased secretion of aldosterone. The physiologic effect of increased renin secretion depends upon levels of renin substrate, converting enzyme and the ability of the adrenal to respond. The last of these is apt to be particularly variable in congenital adrenal hyperplasia. There are also nonrenin dependent renal mechanisms (4) which may have compensated to some degree for sodium depletion.

If sodium-wasting in $\mathrm{CAH}$ is due simply to deficiency of mineralocorticoids, and increased PRA represents the body's at-

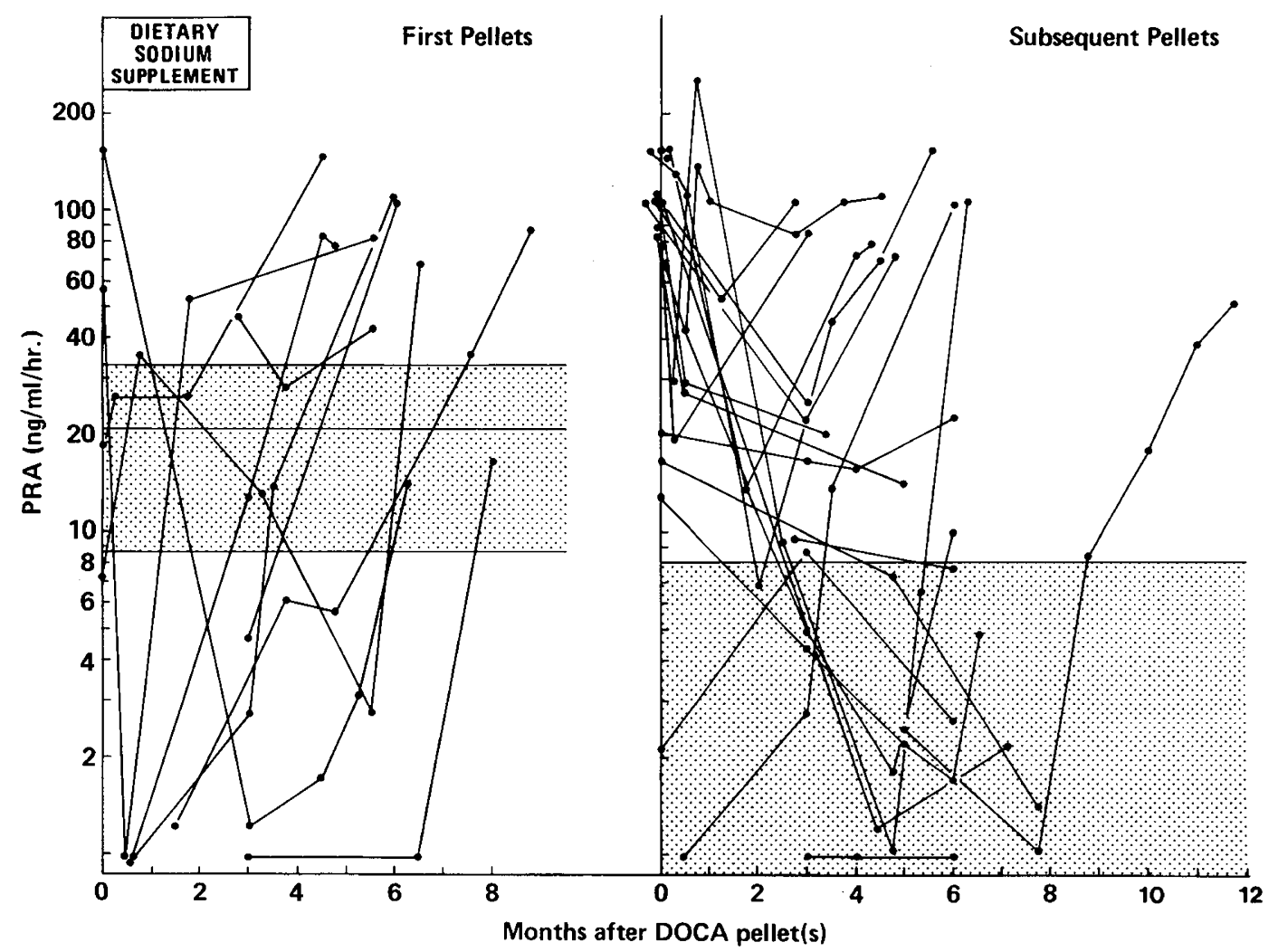

Fig. 2. Serial PRÁ in individual infants at various times after subcutaneous implantation of DOCA pellets are dots connected by lines. Left panel: infants receiving two $125 \mathrm{mg}$ DOCA pellets as initial therapy. Right panel: infants receiving one $125 \mathrm{mg}$ DOCA pellet as subsequent therapy. Each pellet cycle is considered as a separate series of values. Shaded zones: left panel = mean \pm S.D. for normal infants $0-6$ months of age, right panel $=$ normal range for older children and adults. 


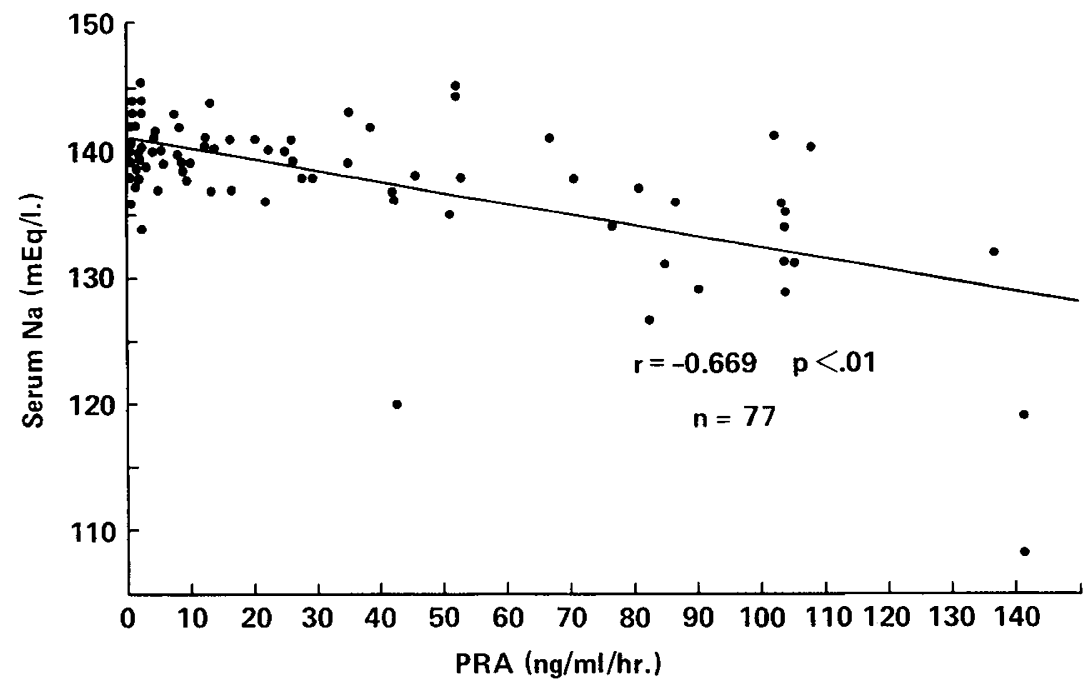

Fig. 3. Serum sodium concentration vs PRA in infants treated with subcutaneous DOCA pellets.

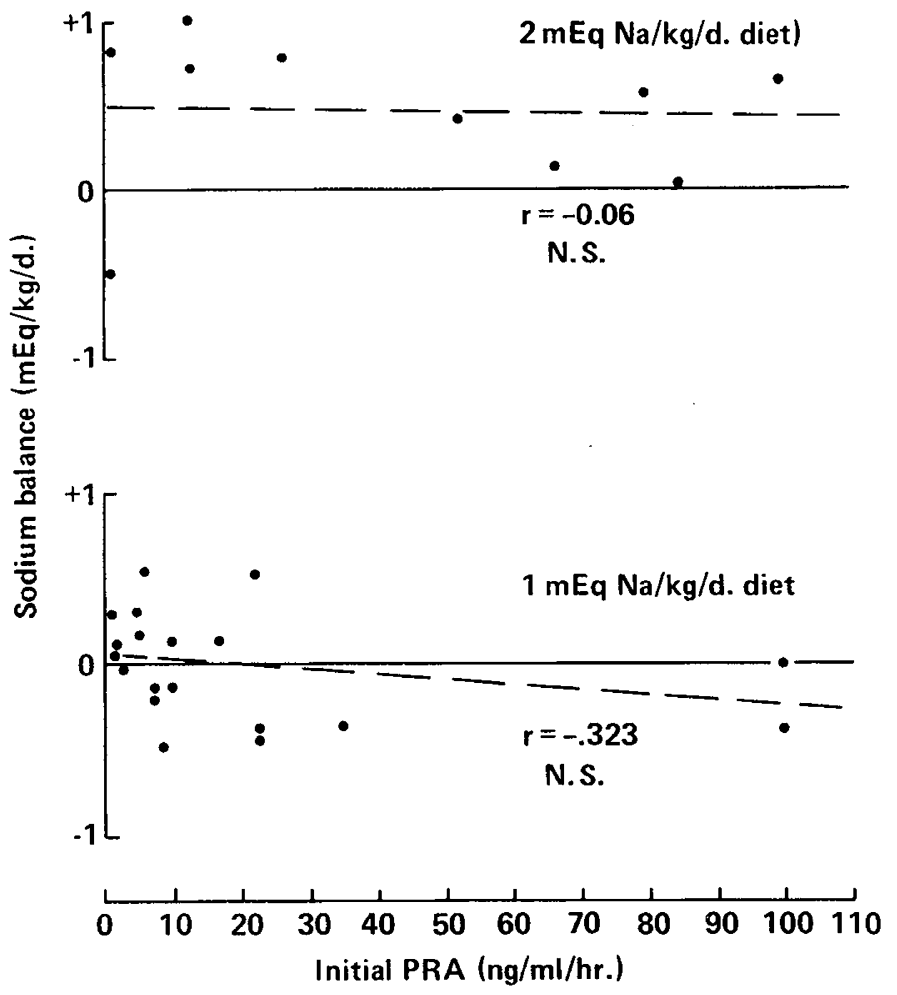

Fig. 4. PRA on admission (initial value) is compared to sodium balance of day 3 of the indicated diet in infants treated with subcutaneous DOCA pellets.

tempt to compensate for sodium depletion, increased PRA should have been associated with increased urinary sodium loss when sodium intake is held at a constant, low level. Such a relationship was not apparent in the infants at 3-6 months post DOCA pellet implantation. Regardless of the fact that during that interval PRA levels were increasing in most subjects, indicating sodium depletion, increased PRA levels at the start of a balance study did not correlate with increased $\mathrm{Fe}_{\mathrm{Na}}$ or with more negative sodium balance. Sodium balance was maintained even with markedly elevated PRA, and these infants demonstrated the ability to reduce $\mathrm{Fe}_{\mathrm{Na}}$ acutely, as well. Thus, they were able to compensate acutely for reduced sodium intake, as well as, for an apparent chronic decrease in the availability of DOCA from the pellets. Whether those adjustments were related to endogenous adrenal steroid production or to nonsteroidal renal mechanisms cannot be ascertained from these data.

It has been demonstrated that a number of subjects with salt- losing $\mathrm{CAH}$, when hyperreninemic, are able to secrete aldosterone in significant quantities $(6,10)$. In subjects without adrenal pathology, it is well known that sodium depletion due to diet or certain diuretics (13) and a reduction in blood volume due to hemorrhage can lead to increased PRA. These changes can be reversed by administration of sufficient sodium or blood. In adrenalectomized dogs (1), sodium depletion occurred along with marked elevation of PRA. Replacement therapy with DOCA reduced PRA nearly to normal, whereas the effect of glucocorticoid therapy was minimal. Elevated PRA in adrenal insufficiency, therefore, reflects insufficient mineralocorticoid secretion.

These studies demonstrated that elevated PRA in salt-losing CAH is indicative of the subject's response to sodium depletion, and is thus suggestive of insufficient mineralocorticoid therapy. Whether the PRA level is proportionate to the degree or the duration of sodium depletion cannot be determined from these data. Studies with diuretic-induced sodium depletion in hypertensive adults, however, indicated good agreement between the level of PRA after 4 days and 4 wk of therapy (16), suggesting that the duration of the stimulus may be less significant than its magnitude. The short term changes in the older subjects of this study indicated considerable individual variation in the PRA response to a given degree of sodium loss. Since the present studies in infants strongly suggested that endogenous factors, other than the DOCA therapy, contributed to sodium homeostasis, it may have been that one factor modifying the levels of PRA attained with a given degree of sodium depletion was the subject's endogenous adrenal and/or renal function. Further investigations will be needed to clarify these relationships.

It is concluded that elevated PRA in salt-losing CAH generally indicates sodium depletion to some degree. Whereas the individual patient may have compensated for this enough to maintain sodium balance, additional mineralocorticoid therapy could be beneficial not only in contributing to sodium homeostasis but possibly to other aspects of this disease. Thus, hyperreninemia has been suggested as a factor leading to increased secretion of ACTHdependent steroids (17). In subjects who are being treated daily with an oral mineralocorticoid, increase in the dosage is appropriate when PRA is elevated. In infants treated with subcutaneous DOCA pellets, increased sodium intake may be sufficient. In those infants, however, if PRA is extremely high $(>100 \mathrm{ng} / \mathrm{ml} / \mathrm{h})$ or hyponatremia is present, implantation of an additional DOCA pellet or a change to oral therapy with $9 \alpha \mathrm{FF}$ may be indicated.

\section{REFERENCES AND NOTES}

1. Berns, A. S., Pluss, R. G., Erikson, A. L., Anderson, R. J., McDonald, K. M., and Schrier, R. W.: Renin-angiotensin system and cardiovascular homeostasis in adrenal insufficiency. Amer. J. Physiol., 233: F509 (1977).

2. Bongiovanni, A. W., Eberlein, W. R., Goldman, A. S., and New, M.: Disorders of adrenal steroid biogenesis. Rec. Prog. Horm. Res., 23: 375 (1967). 
3. Bryan, G. T., Kliman, B., and Bartter, F. C.: Impaired aldosterone production in "salt-losing" congenital adrenal hyperplasia. J. Clin. Invest., 44: 957 (1965).

4. DeWardener, E.: The control of sodium excretion. In: Orloff, J. and R. W Berliner, (Eds.), Handbook of Physiology-Section 8, p. 677 (American Physiological Society, 1973).

5. Holcombe, J. H., Keenan, B. S., and Clayton, G. W.: Plasma 17 $\alpha$-hydroxyprogesterone and aldosterone concentrations in infants and children with congenital adrenal hyperplasia - the role of salt-losing hormones in salt wasting. $\mathbf{J}$. Pediatr. 98: 573 (1981).

6. Horner, J. M., Hintz, R. L., and Luetscher, J. A.: The role of renin and angiotensin in salt-losing $2 \mathrm{l}$-hydroxylase deficient congenital adrenal hyperplasia. J. Clin. Endocrinol. Metab., 48: 776 (1979).

7. Hughes, I. A., and Winter, J. S. D.: 17 $\alpha$-hydroxyprogesterone and plasma renin activity. In: P. A. Lee, et al., Eds., Congenital Adrenal Hyperplasia, p. 141 (Univ, Park Press, Baltimore, MD, 1979).

8. Imai, M., Igarashi, Y., and Sokabe, H.: Plasma renin activity in congenital virilizing adrenal hyperplasia. Pediatrics, 41: 897 (1968).

9. Janoski, A. H.: Naturally occurring adrenal steroids with salt-losing properties: relationship to congenital adrenal hyperplasia. In: P. A. Lee, et al., Eds. Congenital Adrenal Hyperplasia, p. 99 (University Park Press, Baltimore, MD, 1977).

10. Keenan, B. S., Holcombe, J. H., Kirkland, R. T., Potts, V. E., and Clayton, G W.: Sodium homeostasis and aldosterone secretion in salt-losing congenital adrenal hyperplasia. J. Clin. Endocrinol. Metab., 48: 430 (1979).

11. Keenan. B. S., McNeel, R., Barrett, G. N., Holcombe, J. H., Kirkland, R. T., and Clayton, G. W.: Plasma androgens in congenital adrenal hyperplasia: androstenedione concentration as an index of adrenal androgen suppression. J. Lab. Clin. Med., 94: 799 (1979)

12. Kowarski. A. J., Finkelstein, J. W., Spaulding, J. S., Holman, G. H., and Migeon C. J.: Aldosterone secretion rate in congenital adrenal hyperplasia: a discussion of the theories on the pathogenesis of the salt-losing form of the syndrome. $J$. Clin. Invest., 44: 1505 (1965).

13. Laragh, J. H., and Sealy, J. E.: The renin-angiotensin-aldosterone hormona system and regulation of sodium, potassium and blood pressure homeostasis In: Orloff, J. and R. W. Berliner, (Eds.), Handbook of Physiology-Renal Physiology-Section 8, p. 831 (American Physiological Society, 1973).

14. Lipton, H. L., Tan, S. Y., Noth, R., Mulrow, P. J., and Genel, M.: Usefulness of plasma renin activity to monitor mineralocorticoid replacement in salt-losing congenital adrenal hyperplasia. In: P. A. Lee, et al., Eds., Congenital Adrenal Hyperplasia, p. 127 (Univ. Park Press, Baltimore, MD, 1977).

15. New England Nuclear Kit, measures angiotensin I (by the method of Cohen E.L. et al., J. Lab. Clin. Med., 77: 1025 (1971).

16. Nielsen, I., Steiness, E., and Hesse, B.: Acute and long-term salt depletion and $\beta$-blockade: plasma renin activity response and its relation to blood pressure reduction in long-term treatment. Acta Med. Scand., 203: 415 (1978)

17. Rosler, A., Levine, L., Schneider, B., Novogroder, B., and New, M.: The interrelationship of sodium balance, plasma renin activity and ACTH in congenital adrenal hyperplasia. J. Clin. Endocrinol. Metab., 45: 500 (1977).

18. Sassard, J., Loras, B., Poncet, J., Vincent, M., and Gharib, C.: Etude de l'activite' r'enine et de la secretion d'aldosterone dans 7 cas d'hyperplasie surrenalienne par deficit en 21-hydroxylase. Ann. Endocrinol. (Paris), 30: 1822 (1969).

19. The Roche Kit utilizes the incubation conditions described by Sealey, J. E. Moon, C., Laragh, J. H., and Alderman, M., Am. J. Med., 61: 731 (1976).

20. Snedecor, G. W., and Cochran, W. G.: Statistical methods, 6th edition, p. 176 (The Iowa State Univ. Press, Ames, Iowa, 1967).

21. Wilkins, L.: The Diagnosis and Treatment of Endocrine Disorders in Childhood and Adolescence, p. 418 (Charles Thomas, Springfield, ILL, 1965).

22. Winters, R. W. In: R. W. Winters (Ed.). The Body Fluids in Pediatrics, p. 113 (Lille Brown, Boston, MA, 1973).

23. Zurbrugg, R. P.: Congenital adrenal hyperplasia. In: Gardner, L. I., Ed., Endocrine and Genetic Diseases of Childhood and Adolescence, p. 476 (W. B. Saunders, Philadelphia, PA, 1975).

24. This work was supported in part by the following grants: American Heart Association, Texas Affiliate (no. 7155); Children's Clinical Research Center Grant USPH-PR-00188 from NIH; Pediatric Research Training Grant HD07091-02 from NIH; and Children's Nutrition Research Center, US Department of Agriculture 58-7B30-9-60.

25. Requests for reprints should be addressed to: Bruce S. Keenan, M.D., Department of Pediatrics, Baylor College of Medicine, 1200 Moursund, Houston. Texas 77030 .

26. The authors wish to express their appreciation to Mrs. Connie Wallace for her editorial assistance.

27. The present address of John H. Holcombe is: Dept. of Pediatrics, U. of Okla Health Science Ctr., P.O. Box 26901, Oklahoma City, Okla. 73190. Pediatric fellow, Department of Pediatrics, Baylor College of Medicine. Fellowship in memory of Joseph S. Loeb and Polly Meyer Loeb, Ben and Della Epstein Fund for Pediatrics and NIH Training Grant HD07091-02.

28. The present address of Donnie P. Wilson is: Pediatric Endocrinology \& Metabolism, Children's Medical Ctr., 5300 E. Skelly Drive, P.O. Box 35648, Tulsa. Okla. 74135. Pediatric Endocrine Fellow, Dept. of Pediatrics, Baylor College of Medicine. NIH Training Grant HD07091-02.

29. Elaine Potts, Research Dietitician, Clinical Research Center, Texas Children's Hospital, Houston, Texas.

30. It should be noted that the PRA method for the infant studies was different from that for the older subjects, though the correlation between the two methods was excellent (see experimental).

31. Received for publication April 7, 1981.

32. Accepted for publication July 2, 1981 . 